\title{
Analysis of Long Period Fiber Grating using Thermally Tunable Multiport Lattice Model
}

\author{
Jun Kye Bae ${ }^{1,2}$, Jinho Bae ${ }^{3}$, Dongyean $\mathrm{Koh}^{1}$, Sang Hyuck Kim ${ }^{1}$, Namkyoo Park ${ }^{2}$ and Sang Bae Lee ${ }^{1}$ \\ ${ }^{1}$ Photonics Research Center, KIST, Seoul 130-791, Korea, Tel : +82-2-958-5696, Fax: +82-2-958-5709, Email: \\ demian@kist.re.kr \\ ${ }^{2}$ Optical Communication Systems Laboratory, School of EECS, Seoul National University,Seoul 151-744, Korea \\ ${ }^{3}$ College of Ocean Science, Cheju National University, 1 Ara 1-dong, Jeju 690-756, Korea
}

\section{Abstract}

We propose an analysis technique of thermally tunable

LPFG Filter with multiple cladding mode using multiport lattice filter model. Using LPFG with different cladding modes, we could verify the validity of the proposed model experimentally.

\section{Introduction (size 10, Bold)}

In an LPFG, there is one fundamental core mode and multiple cladding modes, which all propagate in the same (forward) direction and LPFG shows a transmission spectrum consisting of district resonant loss peaks. Various LPFG devices have been demonstrated for use as band rejection filters[1,2] and sensors[3]. In all fields of potential application, knowledge of the sensitivity of the LPFG is clearly important and characterization of thermal sensitivity of LPFG is an important precursor to practical device design.

Several analysis methods based on LPFG have been reported, including using a sum of each Gaussian transmission spectrum concatenated by LPFG, 2X2 fundamental matrix model or Gel'fand-LevitanMarchenko[4] coupled equations. Furthermore, and extended fundamental matrix model with thermal changes have been proposed to analyzing the transmission of non-uniformly heated LPFG filter[5]. These methods consider the coupling between a single fundamental core mode and only one of cladding modes. But this independent treatment of each dip is not suitable when the wavelength range is so broad that it includes several dips or when each uniform LPFG section of the concatenated LPFGs has a different grating period (or index) so that each section has a different coupled mode.

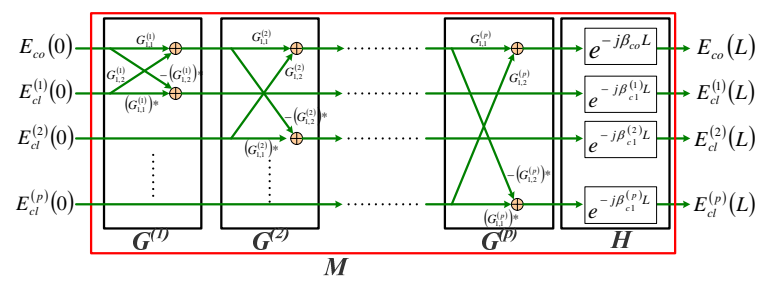

Fig. 1. The multiport lattice filter that models the LPFG in which a core mode couples with multiple cladding modes

In this paper, we analyze the temperature variation of coupling for each cladding modes and develop a transfer matrix which is extension of the $2 \times 2$ fundamental matrix formed by considering the multiple couplings between the core mode and each of the cladding modes simultaneously. To verify the validity of the proposed analysis method, we have fabricated the LPFGs and their experimental results compared with theoretical ones will be presented.

\section{Multiport Lattice Filter Model}

The often-preferred approach to analyzing the coupling between a core mode and one of cladding mode is $2 \times 2$ fundamental matrixes which are derived from Maxwell equations. To analyze multiple couplings between a core mode and multiple cladding modes simultaneously as shown figure 1 , we need to develop an accurate and versatile extension of the conventional 2x2 fundamental matrix which model 
coupling between the core mode and one of the cladding modes. Combing all fundamental matrix for each cladding mode, we can get $\left(p^{+1}\right) \mathrm{x}\left(\mathrm{p}^{+1}\right)$ multiport lattice matrix M of the LPFG as follows [6]

$$
\left[\begin{array}{c}
E_{c o}(L) \\
E_{c l}^{(1)}(L) \\
\vdots \\
E_{c !}^{(p-1)}(L) \\
E_{c l}^{(p)}(L)
\end{array}\right]=M\left[\begin{array}{c}
E_{c 0}(0) \\
E_{c \mid}^{(1)}(0) \\
\vdots \\
E_{c 1}^{(p-1)}(0) \\
E_{c l}^{(p)}(0)
\end{array}\right], \quad M=H G^{(p)} \cdot G^{(p-1)} \cdots G^{(2)} \cdot G^{(1)}
$$

where $G^{(p)}$ is the $(\mathrm{P}+1) \times(\mathrm{P}+1)$ indentity matrix except for the four entries adopted from fundamental matrix of $p^{\text {th }}$ cladding mode as follows :

$$
\begin{aligned}
& G_{1,1}^{(p)}=\left\{\operatorname{COS}\left(S_{p} L\right)-j \frac{\delta_{p}}{S_{p}} \sin \left(S_{p} L\right)\right\} e^{j \delta_{p} L}=\left(G_{p+1, p+1}^{(p)}\right)^{*} \\
& G_{1, p+1}^{(p)}=-j \frac{k_{p}}{S_{p}} \sin \left(S_{p} L\right) e^{j \delta_{p} L}=-\left(G_{p+1,1}^{(p)}\right)^{*}
\end{aligned}
$$

The matrix $\mathrm{H}$ represents the phase shift of the LPFG. $\delta_{\mathrm{p}}$ and $\kappa_{\mathrm{p}}$ are detuning factor and coupling coefficient of $\mathrm{p}^{\text {th }}$ cladding modes, respectively. $S_{p}$ is defined as ,

$$
S_{p}=\sqrt{\kappa_{p}^{2}+\delta_{p}^{2}}
$$

Since, the transfer matrix coefficient $G_{1,1}^{(p)}, G_{1, p+1}^{(p)}$, $G_{p+1,1}^{(p)}, G_{p+1, p+1}^{(p)}$ are the function of detuning factor $\delta_{p}$ and coupling coefficient $\kappa_{p}$ which depend on thermal change, we need to identify the temperature dependent of coupling coefficient $\kappa$ and detuning factor $\delta$ for each order of cladding mode. Using the boundary condition as mentioned in previous report [5], $\kappa$ and $\delta$ can be calculated as shown below.

$$
\begin{aligned}
\kappa(T, \lambda)= & \frac{\pi \Delta n C}{\lambda} \cong \frac{c(T)}{\lambda} \\
\delta(T, \lambda) & =\frac{1}{2}\left(\beta_{c o}-\beta_{c l}^{p}-\frac{2 \pi}{\Lambda}\right) \\
& =\frac{1}{2}\left(\Phi(\lambda)-\Phi\left(\lambda_{\text {res }}\right)\right) \\
& \cong \frac{1}{2} a_{1}(T)\left\{\lambda-\lambda_{\text {res }}(T)\right\}
\end{aligned}
$$

where $\Delta \mathrm{n}$ is the induced index change, $\Phi$ is intermodal dispersion function and $\mathrm{C}$ is the overall integral factor which is assumed to be independent constant to the wavelength, refractive index, grating periods. $\mathrm{c}(\mathrm{T})$ and $\mathrm{a}_{1}(\mathrm{~T})$ are coupling ratio and first coefficient of Taylor expansion for temperature state, respectively. For this definition, $c(T), a_{1}(T)$ and $\lambda_{\text {res }}(T)$ decide the peak depth, peak bandwidth, resonance wavelength for each cladding modes.

To develop c(T) and $a_{1}(T)$, we fabricate the LPFG1

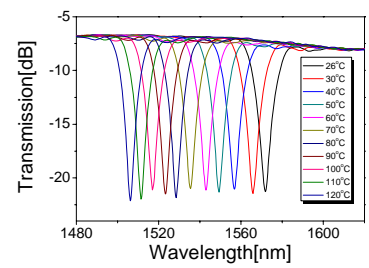

(a)

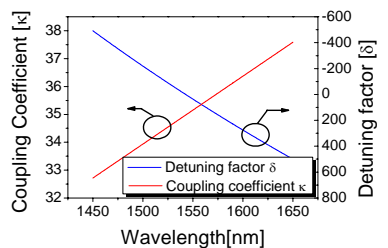

(c)

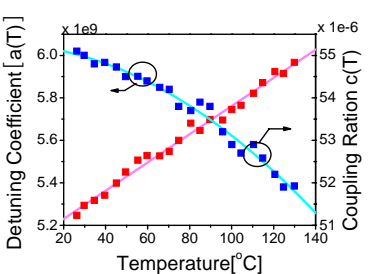

(e)

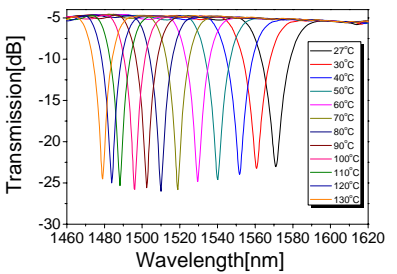

(b)

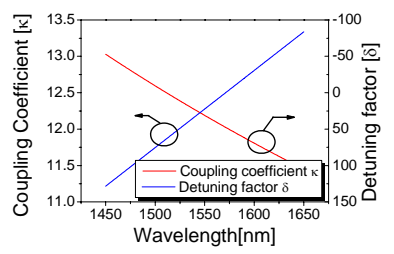

(d)

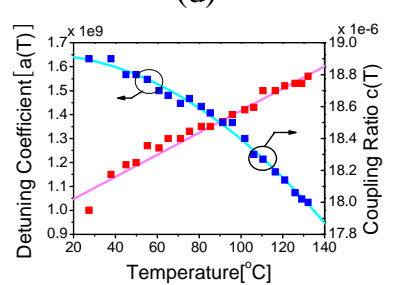

(f)
Fig 2. Transmission spectra, detuning coefficient, coupling ratio with thermal changes and coupling coefficient and detuning factor in room temperature of LPFG 1 (a, c, e) and LPFG 2(b, d, f).

( $\Lambda=356 \mathrm{um}, \quad \mathrm{L}=4 \mathrm{~cm}), \quad \mathrm{LPFG} 2(\Lambda=433 \mathrm{um}, \quad L=5 \mathrm{~cm})$ which have different order of resonance peak to each other in 1500 1600nm range. The measured transmission spectra of each uniform LPFG along the thermal changes $\mathrm{T}=\left(24^{\circ} \mathrm{C} \sim 125^{\circ} \mathrm{C}\right)$ were shown in figure 2(a). The $\kappa$ and the $\delta$ for the spectrum in figure 2(a, b) without thermal change (in our case $\mathrm{T}=26^{\circ} \mathrm{C}$ ) can be calculated as shown in Fig. 2(c, d). The temperature variation $\mathrm{c}(\mathrm{T})$ and $\mathrm{a}(\mathrm{T})$ can be determined by fitting measured transmission spectrum in figure 2(a) and (b)

\section{Experimental result}

A non-uniform LPFG which composed of two uniform LPFGs (LPFG 1 and LPFG 2) which has different cladding modes in 14500 1600nm range was used for verifying the validity of the proposed analysis 
model(LP 05 for LPFG1 and $\mathrm{LP}_{07}$ for LPFG2). The grating period and the length of LPFGs were $356 \mu \mathrm{m}$ and $11 \mathrm{~cm}$ for LPFG1, $433 \mu \mathrm{m}$ and $5 \mathrm{~cm}$ for LPFG2.

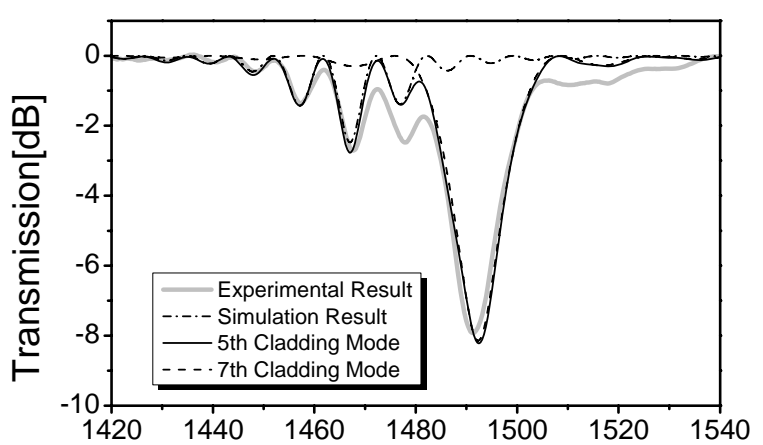

(a)

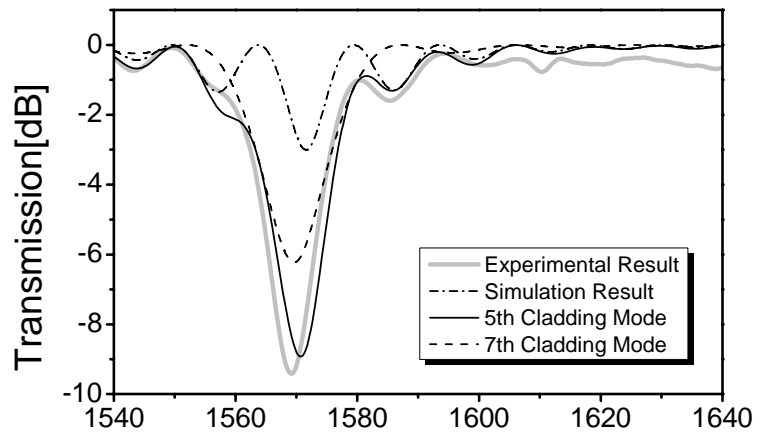

(b)

Fig. 3. Measured and calculated LPFG spectra of the LPFG (a) in room temperature $\left(28^{\circ} \mathrm{C}\right)$ and (b) in $130^{\circ} \mathrm{C}$

The measured and the theoretically calculated spectrum curves are compared in figure 4. Peak wavelength, depth, and spectral shape of LPFG spectrum was changed with the temperature variation as shown figure 3 . The calculated transmission spectra (black thin line) match well with the experimental result (gray thick line) in the wavelength band. Figure 4 shows the $\mathrm{LP}_{05}$ (dash) and $\mathrm{LP}_{07}$ (dash dot) mode distributions for the LPFG spectrum and shows the independent characteristics for each other. As different cladding modes are employed for each LPFG, undesirable recouping and multipath interference effect are suppressed. In this report, we use only two cladding mode to develop and analysis the LPFG spectrum, we can expand the filter model to include every cladding mode of LPFG and can conveniently use this filter model to analysis and synthesis LPFG filter.

\section{Conclusions}

We propose an analysis technique of thermally tunable LPFG Filter with multiple cladding modes using multiport lattice filter structure. By investigating variations of coupling coefficient and detuning factor changed by temperature, we could analysis the variation of the LPFG filter spectrum. Using the LPFG with different cladding modes, we could verify the validity of the proposed model experimentally and we have observed that the transmission spectra calculated using the proposed model are closed to the corresponding measured spectra in the wavelength band of interest.

\section{References}

1. J. Bae et al, IEEE PTL, 17(2005), 1226

2. M. Harurnoto et al, JLT, 20(2002), 1027

3. A. D. Kersey et al, JLT, 15(1997), 1442

4. E. Peral et al, JQE, 32(1996), 2078

5. J. Bae et al, OE, 12(2004), 797

6. J. Bae et al, JLT, 22(2004), 1976 